\title{
Enhanced optical transmission of the triple-layer resonant waveguide structure
}

\author{
I.Ya. Yaremchuk ${ }^{1}$, V.M. Fitio ${ }^{1}$, Ya.V. Bobitski ${ }^{1,2}$ \\ ${ }^{1}$ Lviv Polytechnic National University, Department of Photonics, \\ 12, Bandera str., 79013 Lviv, Ukraine \\ Phone: 8-032-2582581, e-mail: iryna.y.yaremchuk@lpnu.ua \\ ${ }^{2}$ Faculty of Mathematics and Natural Sciences, \\ University of Rzeszow, Pigonia Str. 1, 35959 Rzeszow, Poland
}

\begin{abstract}
In this paper, we propose and demonstrate a novel guided-mode resonant filter based on a metallic grating sandwiched between two dielectric layers for TE polarization. A theoretical model based on rigorous coupled wave analysis has been developed. The transmission spectra of the grating-based structure show a high transmission band with the corresponding amplitude up to $70 \%$ inside the infra-red region. Moreover, the observed optical properties can be exactly tuned by the structural parameters. These properties allow using such structures as compact optical filters, and their spectral characteristics can be easily tuned and scaled.
\end{abstract}

Keywords: filter, transmission, wave-guide resonance.

Manuscript received 19.01.16; revised version received 27.04.16; accepted for publication 08.06.16; published online 06.07.16.

\section{Introduction}

Extraordinary transmission and directional beaming of light through grating structures have attracted much attention due to their fundamental importance for manipulating light at a subwavelength scale [1] and potential applications in optics and photonics [2-4]. Interest in the extraordinary optical transmission process arises due to high available contrast between the metal and the subwavelength apertures. Moreover, the extraordinary optical transmission offers the prospect of a multitude of applications, since remarkably high transmission efficiencies and, concomitantly, high local field enhancements at certain wavelengths can be achieved through the geometry of metal surface [5]. The explanation of the extraordinary optical transmission is still under debate [6-9], although many different theories have been reported to explain this phenomenon [10-13]. It is shown $[14,15]$ that surface electromagnetic modes play a key role in the emergence of the resonant transmission. However, in works [16-18] it was reported that there are two transmission resonances for lamellar transmission metallic gratings: one of them is coupled to the surface plasmon polaritons modes on both the horizontal surfaces of the metallic grating, and the second one is related to the cavity or waveguide modes located inside the slits. However, in scientific literature one can find a lot of researches aimed at using only TM polarization waves and corresponding spectral dependences of the grating transmission. At the same time, the transmission dependence for TE polarization on the thickness and characteristics of the structures can give useful information to explain the anomaly high transmission of such gratings [19-22]. On the other 
hand, the elements exploiting this mechanism can demonstrate novel optical properties and offer new possibilities. Understanding the coupling of waves by metallic nanostructures has fundamental interest and practical importance in designing optical devices that could become important elements for future nano-optical systems. The primary application of such resonant periodic structures are: filters based on a dielectric layer sandwiched between two metal-dielectric gratings [23]; resonant waveguide-metallic subwavelength grating [24]; subwavelength compound metallic grating deposited on the waveguide structure [25]; nano-deep corrugated long-period waveguide gratings [26] etc.

In this work, the intensity of distribution fields inside the slit and on the surfaces of the metal grating sandwiched between two dielectric layers have been researched. This allowed us to explain nature of the resonances and to determine conditions for the high transmission. It has been demonstrated that metallic grating sandwiched between two dielectric layers can give rise to the enhanced transmission phenomena in the infrared region without the surface plasmon-polariton modes. The proposed structure allows enhanced transmission for the TE polarized field in a narrow band of wavelengths. The value and position of the transmission peak strongly depend on the structure geometric parameters.

\section{Theoretical background}

The mechanism of extraordinary optical transmission through grating-based structures is studied by rigorous coupled-wave analysis (RCWA) [27-29]. Specifically, the RCWA and analytical transmission functions based on the same principles $[7,17,30,31]$ are in very good agreement with various experimental results in different configurations. Using the RCWA method to the periodic structure shown in Fig. 1, the dielectric function of materials and electromagnetic fields are expanded in a Fourier series. An infinite series of coupled equations is created when substituting both Fourier series into Maxwell's equations. RCWA reduces the electromagnetic field calculation to an algebraic eigenvalue problem. The problem is described by the system of linear differential equations [32]:

$\frac{d^{2} \mathbf{G}}{d z^{2}}=\mathbf{B}_{1} \mathbf{B}_{2} \mathbf{G}=\mathbf{B}_{e} \mathbf{G}$ for TE polarization,

$\frac{d^{2} \mathbf{F}}{d z^{2}}=\mathbf{B}_{2} \mathbf{B}_{1} \mathbf{F}=\mathbf{B}_{m} \mathbf{F}$ for TM polarization,

where $\mathbf{G}$ and $\mathbf{F}$ are the vectors containing the expansion coefficients of the electric and magnetic fields, respectively; $\quad \mathbf{B}_{1}=-i k_{0} \mathrm{I}+i k_{0}^{-1}\|k\|_{j}\|\varepsilon\|_{j p}^{-1}\|k\|_{j}$, $\mathbf{B}_{2}=-i k_{0}\left\|\frac{1}{\varepsilon}\right\|_{j p}^{-1},\|\varepsilon\|_{j p}$ is the Toeplitz matrix composed of the coefficients of expansion of the grating material dielectric constant in the Fourier complex series, $\mathbf{I}$ - unit matrix, $\|k\|_{j}^{2}$ - diagonal matrix, elements of which equal to $k_{j x}^{2}, k_{j, x}=k_{0, x}-j \frac{2 \pi}{\Lambda}, k_{0, x}=\frac{2 \pi}{\lambda} \sqrt{\varepsilon_{1}} \sin \theta_{1,0}, \theta_{1,0}$ angle of incidence of the plane wave in surrounding medium.

The reader may refer to several papers $[27,28,32]$ describing in detail the mathematical procedure used in this simplified model.

The enhanced transmission can be obtained for the grating with the thickness defined by the expression [19]:

$d_{\max }=d_{11}+d_{13}+(m-1) \Delta d=d_{0}+(m-1) \Delta d$,

where $d_{11}, d_{13}$ are parts of grating thickness that are adjoining to 1 and 3 homogeneous media and are defined by the grating parameters, the wavelength and dielectric constants of the corresponding medium; $m=1,2,3, \ldots ; \Delta d$ is determined by the propagation constant $\beta$ inside the slit, according to the expression $\Delta d=\pi / \operatorname{Re}(\beta)$ [33]. The propagation constant $\beta$ can be expressed according to TE and TM waves as follows:

$$
\begin{aligned}
& D(\beta)=w(u \cos (u)+v \sin (u))-u(u \sin (u)-v \cos (u)), \\
& D(\beta)=\frac{w}{\varepsilon_{21}}\left(\frac{u}{\varepsilon_{22}} \cos (u)+\frac{v}{\varepsilon_{22}} \sin (u)\right)- \\
& -\frac{u}{\varepsilon_{22}}\left(\frac{u}{\varepsilon_{22}} \sin (u)-\frac{v}{\varepsilon_{22}} \cos (u)\right),
\end{aligned}
$$

where $a$ is the attenuation constant, $u=a \sqrt{\varepsilon_{21} k^{2}-\beta^{2}}$, $w=v=i a \sqrt{\varepsilon_{22} k^{2}-\beta^{2}}, \quad k=2 \pi / \lambda$. The waveguide modes with $\beta$ constant, for which $D(\beta)=0$, can be only propagating.

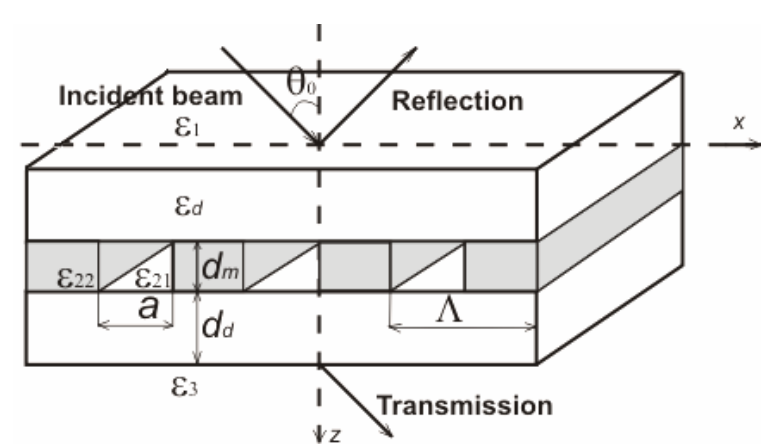

Fig. 1. Geometry of the metallic grating sandwiched between two dielectric layers. 
It should be noted that $\beta$ constant, which has been determined on the base of the equations (3) and (4), is satisfied by the expression:

$-i \sqrt{\mu} \approx \beta$,

where $\mu$ is the eigenvalue of $\mathbf{B}_{e}$ or $\mathbf{B}_{m}$ matrix of the system equation (1). The number of accounted diffraction orders for the diffraction analyses (dimension of matrix $\mathbf{B}_{e}$ or $\mathbf{B}_{m}$ ) determines accuracy of determining the propagation constant.

\section{Results and discussions}

It is known that subwavelength metallic grating covered with a thin dielectric layer shows extraordinary optical transmission under TE polarization. Since, excitation of TE-polarized dielectric waveguide modes inside the dielectric film strongly increases the TE-polarized transmission [34]. We present series of calculations for TE-polarized light being normally incident on the structure with the following grating parameters: $\varepsilon_{22}=9.0, \varepsilon_{21}=\varepsilon_{m}, \Lambda=1.3 \mu \mathrm{m}$, and $a=0.143 \mu \mathrm{m}$ to research the mechanism of the enhanced transmission by the metallic grating sandwiched between two dielectric layers (see Fig. 1). The dielectric constant of dielectric layers is $\varepsilon_{d}=9.0$.

The waveguide effect with low attenuation inside the slit is impossible for such grating parameters. The propagation constant is $\beta=(0.0715-i 11.23) \mu \mathrm{m}^{-1}$ for this slit. Therefore, binary metallic grating with the depth $1.0 \mu \mathrm{m}$ and without dielectrics layers has transmission less than 0.0001 [19]. If the grating is confined by the dielectric layers realizing the waveguide effect, then one can achieve the enhanced transmission (Fig. 2).

Fig. 2a shows dependences of the transmission and reflection for the lowest resonance thickness of the dielectric layers $d_{d}$ at the grating depth $0.2 \mu \mathrm{m}$, the operating wavelength is $1.5 \mu \mathrm{m}$. One can see that significant transmission at a fixed thickness of the dielectric layers is possible. One can also suppose that such high transmission is a result of the waveguide effect in the dielectric layers. The lowest resonance thickness of the dielectric layer depends on the grating depth. Grating depth $d_{m}=0.1 \mu \mathrm{m}$ corresponds to the thickness of dielectric layers $d_{d}=0.1146 \mu \mathrm{m}, d_{m}=0.2 \mu \mathrm{m}$, respectively, to $d_{d}=0.1204 \mu \mathrm{m}$, and $d_{m}=0.3 \mu \mathrm{m}$ to $d_{d}=$ $0.1212 \mu \mathrm{m}$. It must be noted that thicknesses of dielectric layers are equal and designated as $d_{d}$. Thus, the grating depth has only insignificant effect on the resonant thickness of the dielectric layers. This result agrees with the waveguide theory, so far as the phase of the reflected wave at the boundary between dielectric and metal changes a bit with changing the thickness of metallic film within the range $0.1 \ldots 0.3 \mu \mathrm{m}$. The waveguide modes, which are distributed inside the adjacent slits, together do not interact due to the fact that the electromagnetic field penetrates into the metal to a depth of the skin layer. Fig. $2 b$ shows the dependences of transmission and reflection on the grating depth for the thickness of dielectrics layers $0.1204 \mu \mathrm{m}$. The transmission approaches to zero, when the grating depth increases up to $0.6 \mu \mathrm{m}$. On the other hand, reflection doesn't reach unity. The transmission is higher than 0.69 at the grating depth $0.2 \mu \mathrm{m}$.

Fig. 3 shows the dependence of transmission on the wavelength. The transmission has singularity within a narrow spectral band, which can be explained by a resonance.
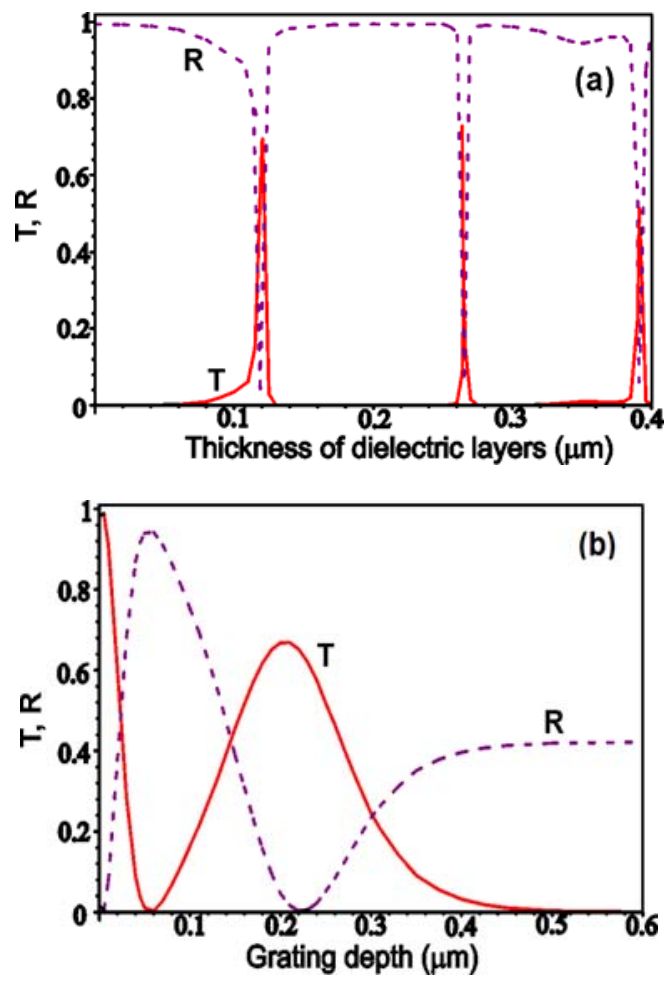

Fig. 2. The calculated dependence of transmission and reflection spectra of the metallic grating.

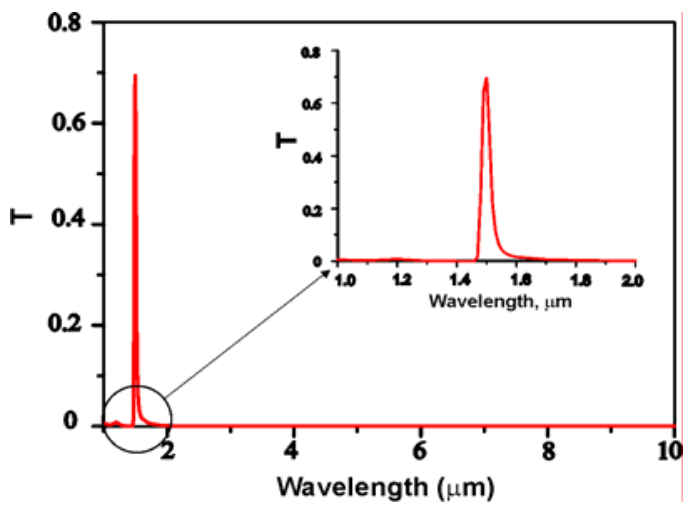

Fig. 3. The calculated transmission spectrum of the metallic grating placed between two dielectric layers with the thickness of dielectric layers $0.1204 \mu \mathrm{m}$ and that of the grating depth $0.2 \mu \mathrm{m}$. 
Distribution of the modulus electric field intensity along the coordinate $x$, i.e., along the grating period, is presented in Fig. 4. The curves 1 and 5 correspond to the field distribution at the boundary of the dielectric layers and the homogeneous media with the dielectric constant $\varepsilon=1.0\left(\varepsilon=\varepsilon_{1}=\varepsilon_{3}\right)$; curves 2 and 4 correspond to the field distribution near the boundary between grating and dielectrics layers; curve 3 corresponds to the field distribution in the middle of the grating placed between two dielectric layers on the thickness of dielectric layers at the constant grating depth $0.2 \mu \mathrm{m}$ (a) and on grating depth at the constant thickness of the dielectric layers $0.1204 \mu \mathrm{m}(\mathrm{b})$.

Fig. 4a presents the calculation results when the imaginary part of dielectric constant of metal was taken into account. The results presented in Fig. $4 \mathrm{~b}$ correspond to the case when imaginary part of the dielectric constant of metal is negligible. One can see that, in the case of neglected imaginary part of dielectric constant of metal, the transmission is equal to unity and the reflection is equal to 0 . There is excellent coincidence of curves 1,5 and 2, 4 for this case.
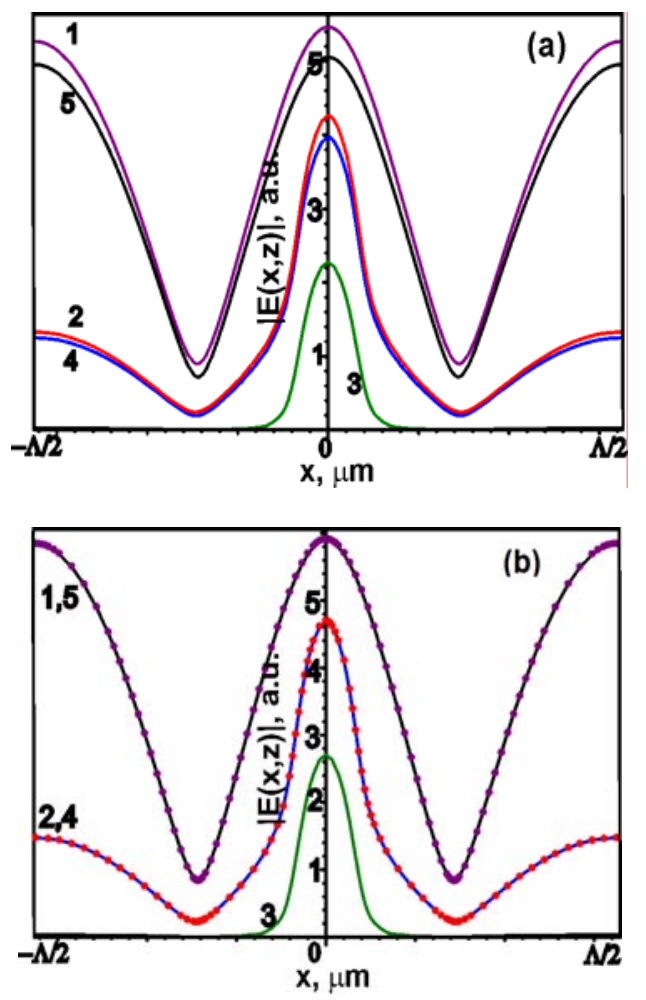

Fig. 4. The distributions of tangential component of the modulus of electric field intensity along the grating period for the thickness of dielectric layers $0.1204 \mu \mathrm{m}$ and that of the grating depth $0.2 \mu \mathrm{m}$ : (a) the imaginary part of the dielectric constant for metal was taken into account in calculations, (b) imaginary part of the dielectric constant for metal is negligible. The points represent curve 4 and 5 in (b).
The amplitudes of the field in arbitrary units are significantly higher than the unity amplitude of the incident wave. Thus, resonance of field occurs in the dielectric layers. On the other hand, the intensity of this resonance is determined by the grating depth. Curves 1 , 5 and 2, 4 are separated with the increase of the grating depth. In this case, the field decreases inside the slit, if the imaginary part of the dielectric constant of metal is taken into account. The resonance is missed inside the slit, since the propagation constant has large imaginary component in the slit.

One can consider that two waveguides (resonators) are interconnected by the slit and the coupling coefficient is determined by the length of slit (grating depth). High transmission is achieved as a result of intense field that is formed by the grating in the dielectric layer over the slit. This mechanism of the enhanced transmission is impossible for TM polarization, since the propagation constant has a insignificant imaginary part even for the slit width much less than the wavelength. Therefore, presence of the dielectric layers only decreases transmission as a result of propagation of the wave under participation of the dielectric layers like that in a waveguide. This gives additional losses of electromagnetic energy caused by the interaction field with the metallic surface.

\section{Conclusions}

The resonant transmission for TE polarization occurs due to the waveguide effect, and there is a minimum width of slit, where the waveguide effect and, consequently, high transmission are possible. Optical transmission of the metallic grating sandwiched between two dielectric layers is found to be surprisingly large at particular wavelengths for TE polarization. Resonant transmission is achieved as a result of the intense field formed by the grating inside the dielectric layer over the slit. The transmission profile can be adapted by adjusting four major parameters: the metallic grating period and thickness, thickness of dielectric layers and refractive indices of grating and dielectric layers. The high transmission of the metallic grating confined between dielectric layers opens up a new dimension in the design and operation of selective narrow-band filters.

\section{Acknowledgments}

The work was supported by Ministry of Education and Science of Ukraine (grant DB $\backslash$ Tekton no 0115U000427).

\section{References}

1. T.W. Ebbesen, H.J. Lezec, H.F. Ghaemi, T. Thio, P.A. Wolff, Extraordinary optical transmission through sub-wavelength hole arrays // Nature, 391(6668), p. 667-669 (1998). 
2. A. Karabchevsky, O. Krasnykov, I. Abdulhalim, B. Hadad, A. Goldner, M. Auslender, S. Hava, Metal grating on a substrate nanostructure for sensor applications // Photonics and Nanostructures - Fundamentals and Applications, 7(4), p. 170-175 (2009).

3. N.C. Lindquist, P. Nagpal, K.M. McPeak, D.J. Norris, S.H. Oh, Engineering metallic nanostructures for plasmonics and nanophotonics // Repts. Prog. Phys. 75(3), 036501(2012).

4. Z. Liu, H. Li, G. Cao, H. Xu, G. Yan, H. Yang, $\mathrm{X} . \mathrm{Xu}$, Tunable optical transmission properties of $\mathrm{Au} / \mathrm{Al}$ multilayer slit gratings // J. Opt. 14(5), 055003 (2012).

5. M.A. Vincenti, D. de Ceglia, M. Scalora, Nonlinear response of GaAs gratings in the extraordinary transmission regime // Opt. Lett. 36(23), p. 46744676 (2011).

6. A. Barbara, P. Quémerais, E. Bustarret, T. LopezRios, Optical transmission through subwavelength metallic gratings // Phys. Rev. B, 66(16), 161403 (2002).

7. M.M.J. Treacy, Dynamical diffraction explanation of the anomalous transmission of light through metallic gratings // Phys. Rev. B, 66(19), 195105 (2002).

8. G. Gay, O. Alloschery, B.V. De Lesegno, J. Weiner, H.J. Lezec, Surface wave generation and propagation on metallic subwavelength structures measured by far-field interferometry. // Phys. Rev. Lett. 96(21), 213901 (2006).

9. J. Wang, Y. Wang, X. Zhang, K. Yang, Y. Wang, S. Liu, Y. Song, Investigation on the transmission of subwavelength metallic cavity arrays // Optik Intern. J. for Light and Electron Optics, 122(18), p. 1650-1653 (2011).

10. L. Martin-Moreno, F.J. Garcia-Vidal, H.J. Lezec, K.M. Pellerin, T. Thio, J.B. Pendry, T.W. Ebbesen, Theory of extraordinary optical transmission through subwavelength hole arrays // Phys. Rev. Lett. 86(6), p. 1114 (2001).

11. D. Pacifici, H.J. Lezec, H.A. Atwater, J. Weiner, Quantitative determination of optical transmission through subwavelength slit arrays in Ag films: Role of surface wave interference and local coupling between adjacent slits // Phys. Rev. B, 77(11), 115411(2008).

12. N. Yao, M. Pu, C. Hu, Z.A. Lai, Z. Zhao, X. Luo, Dynamical modulating the directional excitation of surface plasmons sources // Optik - Intern. J. for Light and Electron Optics, 123(16), p. 1465-1468 (2012).

13. W. Barnes, W. Murray, J. Dintinger, E. Devaux, T.W. Ebbesen, Surface plasmon polaritons and their role in the enhanced transmission of light through periodic arrays of subwavelength holes in a metal film // Phys. Rev. Lett. 92(10), 107401 (2004).
14. H. Liu, P. Lalanne, Microscopic theory of the extraordinary optical transmission // Nature, 452(7188), p. 728-731 (2008).

15. F.J. Garcia-Vidal, L. Martin-Moreno, T.W Ebbesen, L. Kuipers, Light passing through subwavelength apertures // Rev. Mod. Phys. 82(1), p. 729 (2010).

16. J.A. Porto, F.J. Garcia-Vidal, J.B. Pendry, Transmission resonances on metallic gratings with very narrow slits // Phys. Rev. Lett. 83(14), p. 2845 (1999).

17. Q. Cao, P. Lalanne Negative role of surface plasmons in the transmission of metallic gratings with very narrow slits // Phys. Rev. Lett. 88(5), 057403 (2002).

18. H. Lezec, T. Thio, Diffracted evanescent wave model for enhanced and suppressed optical transmission through subwavelength hole arrays // Opt. Exp. 12(16), p. 3629-3651 (2004).

19. V.M. Fitio, Transmissions of Metallic Gratings with Narrow Slots. In: Laser and Fiber-Optical Networks Modeling, 8-th Intern. Conf. on IEEE, June, 2006, p. 113-116.

20. I.Y. Yaremchuk, V.M. Fitio, \& Y.V. Bobitski, High transmission of light through metallic grating limited by dielectric layers // LFNM'2013, p. 74 (2013).

21. E. Moreno, L. Martín-Moreno, F.J. García-Vidal Extraordinary optical transmission without plasmons: the s-polarization case // J. Opt. A: Pure and Appl. Optics, 8(4), p. S94(2006).

22. H. Li, B.C. Liu, TE Polarization extraordinary transmission through metallic grating with subwavelength slits // Procedia Eng. 29, p. 381-385 (2012).

23. W. Liu, G. Hu, H. Zhu, Y. Chen, Waveguide-metal - dielectric bi-layer gratings for reflective filtering and color security // Optik - Intern. J. for Light and Electron Optics, 126(11), p. 1245-1248 (2015).

24. N. Nguyen-Huu, Y.L. Lo, Y.B. Chen, Color filters featuring high transmission efficiency and broad bandwidth based on resonant waveguide-metallic grating // Opt. Communs. 284(10), p. 2473-2479 (2011).

25. R. Hu, Y. Liang, S. Qian, W. Peng, Dual-band bandpass filter based on compound metallic grating waveguide structure // Opt. Communs. 336, p. 110115 (2015).

26. M. Sharma, S. Pal Design and analysis of nanodeep corrugated waveguide grating-based dualresonant filters in visible and infra-red regions // Optik - Intern. J. for Light and Electron Optics, 124(18), p. 3562-3566 (2013).

27. M.G. Moharam, T.K. Gaylord, Rigorous coupled wave analysis of planar-grating diffraction // JOSA, 71(7), p. 811-818 (1981).

28. M.G. Moharam, T.K. Gaylord, E.B. Grann, D.A. Pommet, Formulation for stable and efficient implementation of the rigorous coupled-wave 
analysis of binary gratings // JOSA A, 12(5), p. 1068-1076 (1995).

29. L. Li, Formulation and comparison of two recursive matrix algorithms for modeling layered diffraction gratings // JOSA A, 13(5), p. 1024-1035 (1996).

30. P. Lalanne, J.P. Hugonin, S. Astilean, M. Palamaru, One-mode model and Airy-like formulae for one-dimensional metallic gratings // $\mathrm{J}$. Opt. A: Pure and Appl. Opt. 2(1), p. 48 (2000).

31. A. Benabbas, V. Halté, J.Y. Bigot, Analytical model of the optical response of periodically structured metallic films // Optics Exp. 13(22), p. $8730-8745$ (2005).
32. I. Yaremchuk, T. Tamulevičius, V. Fitio, I. Gražulevičiūte, Y. Bobitski, S. Tamulevičius, Numerical implementation of the S-matrix algorithm for modeling of relief diffraction gratings // J. Modern Opt. 60(20), p. 1781-1788 (2013).

33. H.G. Unger, Planar Optical Waveguides and Fibres // Oxford Eng. Science Series, Oxford, Clarendon Press, p. 1 (1977).

34. M. Guillaumée, A.Y. Nikitin, M.J.K. Klein, L.A. Dunbar, V. Spassov, R. Eckert, R.P. Stanley, Observation of enhanced transmission for $\mathrm{s}-$ polarized light through a subwavelength slit // Optics Exp. 18(9), p. $9722-9727$ (2010). 